\title{
P300 Event-Related Potentials Mediate the Relationship Between Child Physical Abuse and Externalizing Behavior
}

\author{
Naixue Cui 1,2, Adrian Raine ${ }^{3}$, Cynthia A. Connolly2, Therese S. Richmond ${ }^{4}$, \\ Alexandra L. Hanlon ${ }^{5}$, Catherine C. McDonald ${ }^{2}$ and Jianghong Liu2*
}

${ }^{1}$ School of Nursing and Rehabilitation, Shandong University, Jinan, China, ${ }^{2}$ Department of Family and Community Health, School of Nursing, University of Pennsylvania, Philadelphia, PA, United States, ${ }^{3}$ Department of Criminology, Psychiatry and Psychology, School of Arts \& Sciences, University of Pennsylvania, Philadelphia, PA, United States, ${ }^{4}$ Department of Biobehavioral Health Sciences, School of Nursing, University of Pennsylvania, Philadelphia, PA, United States, ${ }^{5}$ Department of Statistics, Virginia Tech, Blacksburg, VA, United States

\section{OPEN ACCESS}

Edited by: Leonardo De Pascalis, University of Liverpool, United Kingdom

Reviewed by:

Antonia Cascales-Martinez, University of Murcia, Spain Pamela Schuetze, University at Buffalo, United States Xiangru Zhu,

Henan University, China

*Correspondence: Jianghong Liu jhliu@nursing.upenn.edu

Specialty section: This article was submitted to

Developmental Psychology, a section of the journal Frontiers in Psychology

Received: 03 June 2021 Accepted: 05 October 2021 Published: 01 November 2021

Citation:

Cui N, Raine A, Connolly CA, Richmond TS, Hanlon AL, McDonald CC and Liu J (2021) P300 Event-Related Potentials Mediate the Relationship Between Child Physical Abuse and Externalizing Behavior. Front. Psychol. 12:720094. doi: 10.3389/fpsyg.2021.720094
The psychophysiological mechanism linking early childhood experiences to behavior problems remains unclear. This study aimed to examine the association of child physical abuse with P300 event-related potentials (ERP), and to test the mediating effect of P300 amplitude and latency in the relationship between child physical abuse and externalizing behaviors. Cross-sectional secondary data were obtained from 155 children (55.5\% boys, mean age: $11.28 \pm 0.57$ years) who participated in the China Jintan Child Cohort Study. Children self-reported maternal and paternal physical abuse and externalizing behaviors, as well as P300 were obtained in 2013. Additionally, parents and teachers reported child externalizing behaviors in preschool in 2007. P300 were recorded during a standard novel auditory oddball task. Path analysis shows that after controlling for child sex, socioeconomic status, area of residence, $I Q$, and child externalizing behavior in preschool, children exposed to maternal physical abuse exhibited increased novelty P300 amplitude, which links to more externalizing behavior. Novelty P300 amplitude partially mediated the relationship between maternal physical abuse and externalizing behavior. These findings are the first to document the partial mediating effect of P300 amplitude on the abuse-externalizing relationship and are consistent with the view that physical abuse affects the attention bias to novel cues that likely places them at increased risk for the development and maintenance of externalizing behavior.

\section{Keywords: physical abuse, P300 amplitude, event-related potential, externalizing behavior, mediation}

\section{INTRODUCTION}

The relationship between child physical abuse and behavior problems has been well documented in the literature across cultures (Fry et al., 2012; Gershoff et al., 2012; Pace et al., 2019). Consistently, a recent meta-analytic study of 42 studies in Mainland China also found that physically abused children manifested more externalizing behaviors (Cui and Liu, 2020). The link remains significant even after controlling for other forms of maltreatment (Renner and Boel-Studt, 2017; Muniz et al., 2019), and the effect of physical abuse practiced by mothers demonstrates more salient effect on externalizing behaviors than that practiced by fathers (Cui et al., 2018). 
Nevertheless, not all maltreated children develop behavior problems, which raises the question of the mechanism underlying the association between child physical abuse and behavior problems. Altered neurocognitive development related to child physical abuse is suggested to be a potential pathway (McCrory et al., 2012; Kavanaugh et al., 2017), which is supported by the empirical evidence of the mediating effect of neurocognition measured by neuropsychological tasks or functional magnetic resonance imaging (fMRI) in the relationship between child maltreatment and externalizing behavior (Xing et al., 2018; Hallowell et al., 2019).

At the neurophysiological level, the P300 event-related potential (ERP) is a widely-used proxy measure of the allocation of neural resources and neurocognitive processing capability (Polich, 2007). The P300 is a large positive-going peak occurring approximately 300 to $800 \mathrm{~ms}$ following stimulus onset. A threestimulus oddball paradigm is widely used for elicitation of P300, in which participants are instructed to detect infrequent deviant stimuli (target; e.g., low-pitched tone) amongst a series of standard stimuli (non-target; e.g., high-pitched tone) and novel stimuli (e.g., dog barks and bird chirp) (Gao et al., 2011). Two P300 components, the "P3a" and "P3b" can be derived from such a task to assess the participants' neural processes of directing attention to events of importance. The P3a is elicited by novel stimuli (i.e., stimuli with low probability, task-irrelevant, but contextual salience) and is commonly regarded as reflecting a bottom-up process of attention orienting to prepare the organism for deviant events in the environment (Debener et al., 2002; Polich, 2007). The P3b peaks 60-80 ms later than the P3a, and is considered a measure of effortful top-down attentional shift to infrequent but task-relevant stimulus and working memory updating when participants are actively engaged in the task of detecting the targets (Polich, 2007). P300 has been extensively studied in relation to both child maltreatment and externalizing behavior, respectively.

A number of studies have examined the effects of maltreatment on neural processes utilizing ERP. For example, Pollak et al. (2001) have conducted a series of studies on school-age maltreated children and found that maltreated children show hyper-responsive to angry facial expressions and greater P3b amplitude in response to angry facial affect (Pollak and Tolley-schell, 2003; Shackman et al., 2007; Shackman and Pollak, 2014). These findings indicate that enhancement in P3b amplitude may be an adaptive mechanism to help maltreated children to detect and respond to anger stimuli more efficiently (Shackman and Pollak, 2014).

Literature also documents relatively consistent evidence that an attenuated P300 amplitude is an endophenotype of externalizing disorders featured by excessive impulsivity (Patrick et al., 2006; Brennan and Baskin-Sommers, 2018), such as alcohol use disorder and substance disorders (Iacono and Mcgue, 2002; Euser et al., 2012; Hamidovic and Wang, 2019), antisocial behavior and impulsive-antisocial psychopathy (Pasion et al., 2018), attention deficit hyperactivity disorder (ADHD; Bitter, 2011; Kallen et al., 2020), and conduct disorder (Iacono and Mcgue, 2002). Reduced novelty P300 amplitude in oddball tasks using non-affective auditory or visual stimuli was found among criminal psychopaths (Venables and Patrick, 2014), offenders (Brazil et al., 2012), and men convicted of spousal/partner abuse (Stanford and Kockler, 2007) in comparison to healthy controls. P300 amplitude reduction also shows association with deficient inhibit control, a cognitive process that links to externalizing behavior.

Despite the cumulative evidence of the pairwise relationships among child maltreatment, P300, and externalizing behavior, the more complex relationships among the three constructs have not been fully examined. To our best knowledge, only two studies (Shackman et al., 2007; Shackman and Pollak, 2014) have hypothesized the pathway leading maltreatment to behavior problems through impaired neural activity indicated by P300 abnormalities and tested the hypothesis empirically. Shackman et al. (2007) in a study of 30 male and female children reported that physically abused children exhibited increased P300 amplitude to threatening stimuli (i.e., their mother's angry faces and angry voices), and that enhanced P300 amplitude mediated the relationship between physical abuse and child selfreported anxiety. Findings from a subsequent study of 50 boys by Shackman and Pollak (2014) found that physical abuse was associated with increased P300 amplitude to negative visual stimuli (i.e., angry adult faces), but that increased P300 amplitude did not significantly correlate with child self-reported aggression.

The inconsistent findings in these studies may be attributed to the differences in participant characteristics (e.g., both boys and girls vs. boys only and age differences), task modality (both visual and vocal stimuli from children's mothers vs. visual stimuli from unfamiliar adults), and behavioral outcomes (anxiety reported by parents using a questionnaire vs. aggression measured objectively using an aggression task). Moreover, the non-significant finding in Shackman and Pollak (2014) may be due to lack of statistical power since the sample size was small $(N=50)$. In addition, neither study controlled for possible confounders, such as prior behavior problems in the analysis. Therefore, larger-scale studies using a standard task protocol to elicit P300 are necessary to further investigate the relationships among physical abuse, P300, and child externalizing behavior.

The objectives of this study were twofold: (1) to test the relationship between child physical abuse and P300 amplitude elicited by a standard novel auditory oddball task stimuli, and (2) to examine the mediating effect of P300 amplitude to novel and target stimuli in the relationship between child physical abuse and externalizing behavior. Because prior studies have not controlled for the possibility that earlier externalizing behavior could result in later enhanced P300 (as opposed to enhanced P300 predisposing to externalizing behavior), we controlled for externalizing behavior collected 6 years prior to P300 assessment.

\section{MATERIALS AND METHODS}

\section{Design and Participants}

This is a cross-sectional study using secondary data (deidentified) collected from a sub-cohort of children during Wave II (T2) of the China Jintan Child Cohort Study (the Jintan Study). 
The Jintan Study is an ongoing prospective longitudinal study to investigate the impact of environmental exposures, such as lead, on children's neurobehavioral outcomes (Liu et al., 2010, 2015), and child physical abuse was measured as an important social confounder. It initially recruited three sub-cohorts of children when they were 3 to 5 years old in 2004-2005. All of these children were invited to participate in two waves of data collection when they were about 6 years old in preschool in 2005 (sub-cohort 1), 2006 (sub-cohort 2), and 2007 (sub-cohort 3), and when they were about 12 years old in grade 6 elementary school in 2011 (sub-cohort 1), 2012 (sub-cohort 2), and 2013 (sub-cohort $3)$, respectively. The cohort children were regarded representative of children of the same age in Jintan City, a small-scale city on the east coast in Mainland China. Details of the cohort design and sampling information are described elsewhere (Liu et al., 2010, 2011, 2015).

In 2013, in additional to the T2 questionnaire survey, all the children of sub-cohort $3(n=414)$ were also invited to participate in psychophysiological recordings. Out of the 414 children, 155 with complete data on child physical abuse, P300, and externalizing behavior at T2 were included in this study. Comparisons of these children and the remaining sub-cohort children who were not included in this study demonstrated no significant differences in age, sex, socioeconomic status, area of residence, externalizing behaviors at age 6 and 12, and maternal and paternal physical abuse experiences. Table 1 displays the sample characteristics and the comparison results.

We obtained verbal assent from children and informed consent from their parents. This study was approved by the Institutional Review Board (IRB) of the University of Pennsylvania and the Ethics Committee of the Jintan Hospital. Clear instruction of voluntary participation, rights of withdrawing or skipping questions whenever they did not feel like answering was given to the children. Considering the potential distress caused by completing the survey, especially questions regarding harsh parenting practice, information of school psychological services and local professional mental health institutions were provided for all participated children in case they needed. When the study was conducted, there was no mechanism/laws/regulations of reporting and dealing with cases of physical abuse that did not meet the criteria of crime in Mainland China. Therefore, action of reporting was not taken.

\section{Measures \\ Child Physical Abuse at Age 12 Years Old}

Children reported their physical abuse experiences in the previous year using the severe physical assault subscale of the Chinese Version of the Parent-Child Conflict Tactics Scale (CTSPC; Straus and Hamby, 1998). They were asked to provide information on whether they were: (1) hit on body parts besides the bottom with objects, (2) thrown or knocked down, (3) hit with a fist or kicked hard, (4) beaten up, (5) grabbed around the neck and choked, (6) burned or scalded on purpose, or (7) threatened with a knife or other weapons by their mothers and fathers separately in the preceding year $(0=$ "No", or $1=$ "Yes"). Children who answered "Yes" to at least one of these items were regarded as having experienced physical abuse. The CTSPC has shown good construct validity (Straus and Hamby, 1998) and reliability in Chinese studies (Chan, 2012; Cui et al., 2016, 2018). In the present study, Cronbach's alpha coefficients for maternal (0.84) and paternal (0.87) physical abuse were acceptable. Our previous study using the same data source demonstrated that children with only maternal physical abuse scored higher on behavior problems but those with only paternal physical abuse did not, and the effect of both maternal and paternal physical abuse on behavior problems was driven by maternal physical abuse. Therefore, we tested the effect of maternal and paternal physical abuse separately, even though they could co-occur.

\section{Child Externalizing Behaviors at Age 12 and 6 Years Old}

Externalizing behaviors at ages 12 and 6 were reported by children themselves using the Chinese version of the Youth SelfReport (YSR) and by mothers using the Chinese version of Child Behavior Checklist for age 1.5-5 (CBCL/1.5-5), respectively. Each item was evaluated on a 3-point scale (2: "often true", 1: "sometimes", and 0: "not true"). The sum score of all items in the externalizing behavior subscale were normalized (mean $=50.00$ and standard deviation $=10.00)$ to obtain $T$ scores for data analysis according to the measurement manuals (Achenbach and Rescorla, 2000). Higher $T$ scores indicate more externalizing behavior. The YSR and CBCL showed good reliability and validity in Chinese children and adolescents (Su et al., 1998; Wang et al., 2013). Complete data on externalizing behavior at age 12 and 6 were obtained from 279 children and 350 mothers, respectively.

\section{Psychophysiological Assessment at Age 12 Years Old Standard novel auditory oddball task}

Given the overarching goal of the parent study of investigating the impact and mechanism of environmental exposure on child neurobehavioral outcomes, the standard novel auditory oddball paradigm was chosen because it was widely used in studies of brain function and behavioral problems with externalizing traits [e.g., substance abuse (Euser et al., 2012; Hamidovic and Wang, 2019), and psychopathology (Gao et al., 2018)]. The oddball task used in this study contains 280 high-pitched tones (nontarget, presented at $1000 \mathrm{~Hz}$ ) and 35 low-pitched tones (target, presented at $500 \mathrm{~Hz}$ ), as well as 35 novel tones (e.g., dog-bark, bell, bird, and honk) at $75 \mathrm{~dB}$. Each tone lasted for $150 \mathrm{~ms}$, with an inter-stimulus interval of $1.1 \mathrm{~s}$, an inter-trial interval of $1.25 \mathrm{~s}$, and rise and fall times of $5 \mathrm{~ms}$. The target, non-target, and novel tones were presented in random order. The duration of the task was $7.5 \mathrm{~min}$.

Children were tested in a temperature-controlled, light- and sound-attenuated laboratory, with a computer screen placed at a distance of $1 \mathrm{~m}$. For the duration of the task, the children were instructed to keep their eyes fixated on an " $\mathrm{X}$ " on the computer screen. To ensure they could distinguish between the non-target and target tones before the actual test, they were given six practice trials. In the actual test, they were instructed to press a response button as quickly as possible with their dominant hand in response to the 
TABLE 1 | Sociodemographic characteristics of the present sample and comparisons with the children not included in the present study.

\begin{tabular}{|c|c|c|c|c|}
\hline & $\begin{array}{l}\text { Children included }(n=155) \\
\qquad \mathrm{M} \pm \mathrm{SD} / n(\%)\end{array}$ & $\begin{array}{l}\text { Children excluded }(n=259) \\
\qquad \mathrm{M} \pm \mathrm{SD} / n(\%)\end{array}$ & $t / \chi^{2}$ & $p$-Value \\
\hline Age $\left(n_{\mathrm{i}}=155, n_{\mathrm{e}}=257\right)$ & $11.28 \pm 0.57$ & $11.32 \pm 0.54$ & 0.71 & 0.48 \\
\hline Gender & & & 0.01 & 0.98 \\
\hline Girls & $69(44.5)$ & $115(44.4)$ & & \\
\hline Boys & $86(55.5)$ & $144(55.6)$ & & \\
\hline Family location & & & 0.20 & 0.91 \\
\hline Urban & $66(42.6)$ & $113(43.6)$ & & \\
\hline Suburban & $65(41.9)$ & $110(42.5)$ & & \\
\hline Rural & $24(15.4)$ & $36(13.9)$ & & \\
\hline $\operatorname{SES}\left(n_{\mathrm{i}}=133, n_{\mathrm{e}}=140\right)$ & $0.19 \pm 0.99$ & $0.22 \pm 0.98$ & 0.24 & 0.81 \\
\hline $\mathrm{IQ}\left(n_{\mathrm{i}}=113, n_{\mathrm{e}}=91\right)$ & $105.65 \pm 11.39$ & $103.40 \pm 13.09$ & 1.31 & 0.19 \\
\hline Externalizing behavior at age $12\left(n_{\mathrm{i}}=155, n_{\mathrm{e}}=184\right)$ & $52.52 \pm 11.43$ & $51.18 \pm 9.67$ & 1.11 & 0.27 \\
\hline Externalizing behavior at age $6\left(n_{i}=132, n_{e}=218\right)$ & $13.23 \pm 6.74$ & $13.47 \pm 6.96$ & 0.31 & 0.76 \\
\hline Maternal physical abuse & & & 2.52 & 0.11 \\
\hline Yes & $51(32.9)$ & $37(24.7)$ & & \\
\hline No & $104(67.10)$ & $113(75.3)$ & & \\
\hline Paternal physical abuse & & & 1.24 & 0.27 \\
\hline Yes & $54(37.8)$ & $47(29.0)$ & & \\
\hline No & $101(65.2)$ & $115(71.0)$ & & \\
\hline
\end{tabular}

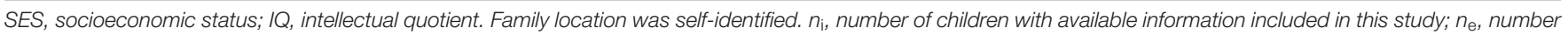
of children with available information but not included in this study.

target tones, but not to other tones. The number and reaction time of correct responses to target, and the number of incorrect responses to non-target (commission error) and novel stimuli (false alarms) were recorded as behavioral informationprocessing indicators.

\section{Event-related potentials recording and data acquisition}

During the oddball task, electroencephalography (EEG) was recorded from an Electro-Cap (Eaton, $\mathrm{OH}$, United States) with tin (Sn) electrodes placed at 12 sites on the scalp (FP1, FP2, F3, F4, F7, F8, P3, P4, T3, T4, O1, and O2) according to the International 10-20 system. A single-channel EEG100C biopotential module (BIOPAC Systems, Inc., Goleta, CA, United States) was used to amplify the EEG signal from each electrode. The same parameters used in Rudo-Hutt's (2014) study were applied. Specifically, the EEG signal was grounded via $8 \mathrm{~mm}$ diameter silver/silver chloride $(\mathrm{Ag} / \mathrm{AgCl})$ electrodes attached to the distal phalanges of the first and second fingers of the non-dominant hand. In addition, an electrooculograph (EOG) channel monitored vertical eye movement via $4 \mathrm{~mm}$ diameter $\mathrm{Ag} / \mathrm{AgCl}$ electrodes placed above and below the supra- and infra-orbital ridges of the left eye. A Q-tip stick was used to abrade the scalp electrode sites, whereas skin on the earlobes and around the left eye was prepared using NuPrep abrasive skin prepping paste. Biopac isotonic recording gel was used as the electrolyte medium for EOG, and Electro-gel was used for the earlobes and scalp. Impedance for EEG was kept below $10 \mathrm{k} \Omega$ and was under $5 \mathrm{k} \Omega$ for most participants, while impedance for EOG and ear electrodes was kept below $20 \mathrm{k} \Omega$. Data from EEG channels were recorded using a bandpass of $0.01-35 \mathrm{~Hz}$ and a $50 \mathrm{~Hz}$ notch filter, with a $1000 \mathrm{~Hz}$ sampling rate and gain set to 5000. Data from the EOG channel were recording using a bandpass of $0.05-35 \mathrm{~Hz}$ and a $50 \mathrm{~Hz}$ notch filter, with a $1000 \mathrm{~Hz}$ sampling rate and gain set to 1000 .

After ERP recording, data from each EEG channel were visually inspected in AcqKnowledge (BIOPAC Systems Inc., Goleta, CA, United States), and clearly artifactual data on EEG (e.g., due to equipment failure or eye movement) were discarded. To help better ensure fidelity of data, the EEG was further processed for remaining artifacts by rejecting EEG epochs that exceeded $\pm 80 \mu \mathrm{V}$ using custom scripts in MATLAB (MathWorks Inc., Natick, MA, United States). Next, the cleaned EEG data was divided into epochs based on stimulus presentation (from $200 \mathrm{~ms}$ before to $800 \mathrm{~ms}$ after each stimulus) and averaged over all trials and all electrodes for each stimulus type in MATLAB to generate the average P300 (i.e., the greatest positive deflection poststimulus) amplitude across electrode sites to target, non-target, and novel stimuli, respectively. P300 was defined as the largest positive-going wave in the range of 100-600 ms after the stimulus onset. A total of 166 children completed the ERP recording.

\section{Other Covariates}

In addition to mother-reported externalizing behavior at age 6, other covariates include child sex, socioeconomic status (SES), area of residence (i.e., urban, suburban and rural areas reported by mothers) at the time of cohort recruitment, and intellectual functioning (IQ) at age 12 because these variables were both associated with physical abuse and externalizing behaviors based on literature (Fergusson and Horwood, 2002; Liao et al., 2011; Cui and Liu, 2016) and data availability of the parent study. SES was calculated as the standardized $z$ score of the sum of standardized $z$ scores of mothers' and father's education years and monthly wage as described in Straus (2004). IQ was measured using the validated Chinese version of the Wechsler 
intelligence scale for children-revised (WISC-R; Dan and Yu, 1990; Liu and Lynn, 2015).

\section{Statistical Analysis}

Complete data were obtained on key variables (physical abuse, P300, and externalizing behavior at age 12 years old) from 159 children. Four children were further excluded because one had an IQ lower than 70 and three had 0 correct responses to targets in the oddball task. Therefore, data from 155 children were used in further analysis.

Sample characteristics of the 155 children were compared with their counterparts who were not included. Bivariate analyses, including independent $t$-tests, Wilcoxon sum rank test, Pearson correlation were used to examine the bivariate association among physical abuse, externalizing behavior, behavioral performances on the oddball task, and target P300 (P3b) and novelty P300 (P3a) amplitude. The P300 variables that showed bivariate relationships with physical abuse or externalizing behavior with $p$-values less than 0.25 were submitted to the path analysis as potential mediators (Bursac et al., 2008).

Path analysis using structural equation modeling (SEM) was implemented to analyze the mediating effect of P300 amplitude based on the model shown in Figure 1. Maternal and paternal physical abuse served as the initial exogenous variables with direct paths to externalizing behavior and indirect paths to externalizing behavior through the P300 variable(s) identified from the above process, controlling for the covariates. The full information maximum likelihood method was used to address missing data on mother-reported externalizing behavior at age 6 . Because a mediation model is saturated, the commonly used goodness of fit indices, such as root square error of approximation (RMSEA), comparative fit index (CFI) and Tucker-Lewis Index (TFI) cannot be applied. Instead, as recommended by Kenny (2016), Akaike Information Criterion (AIC) and Bayesian Information Criterion (BIC) were used to evaluate the mediation model fit by comparing the model without the direct path, the model without the path from predictor to mediator, and the model without the path from mediator to the outcome, respectively. The model with the smallest AIC or BIC was selected. Bootstrapping with 500 replications was used to estimate the bias-corrected 95\% confidence interval for the indirect, direct and total effects. Significance level was set at $\alpha=0.05$. Analyses were performed using STATA 13.0 for Windows (College Station, TX, United States).

\section{RESULTS}

\section{Sample Characteristics}

There were slightly more boys (86, 55.5\%) than girls (69, $44.5 \%)$ among the included sample. The average age was $11.28 \pm 0.57$ years old. A total of $51(32.9 \%)$ children reported maternal physical abuse, and 54 (37.8\%) reported paternal physical abuse, out of whom 41 reported physical abuse by both mothers and fathers. See Table 1.

\section{Bivariate Associations of Physical Abuse With Behavioral Performance on the Oddball Task, P300 and Externalizing Behavior}

Children who experienced physical abuse by either mother or father displayed significantly more externalizing behavior at age 12 when compared with their non-abused counterparts (Table 2). Children with maternal physical abuse showed increased novelty P300 amplitude $(11.40 \pm 4.30 \mu \mathrm{V})$ compared with non-maternal-abused counterparts $(9.63 \pm 4.47 \mu \mathrm{V})$, but the associations of paternal physical abuse with $\mathrm{P} 300$ parameters did not yield any statistical significance. Neither maternal nor paternal physical abuse showed significant associations with the behavioral performances on the oddball task.

\section{Relationship Between Externalizing Behavior at 12 and 6 Years old and P300}

Externalizing behavior at 12 and 6 years old were positively correlated with a small correlation coefficient $(r=0.204$, $p=0.020)$. More externalizing behavior at 12 years old was positively correlated with higher novelty P300 amplitude $(r=0.255, p<0.01)$, but was not significantly correlated with target P300 amplitude $(r=0.028, p=0.728)$. See Table 3. Therefore, novelty P300 amplitude was submitted to path analysis as a mediator and target P300 amplitude was not. Paternal physical abuse was dropped from the path model because it was significantly related to neither the mediator (i.e., novelty P300 amplitude) nor the externalizing behavior.

\section{Path Analysis Results}

The path coefficients of the final mediation model are displayed in Figure 2. The indirect, direct and total effects with biascorrected 95\% confidence interval using the bootstrapping method were 1.08 (0.26-2.58), 4.11 (0.17-10.08), and 5.19 (1.22$11.00)$, respectively. The AIC and BIC values were 5004.666 and 5169.011, respectively. Next, the final model was compared with itself without the direct path $(\mathrm{AIC}=5007.294$, BIC $=5169.595)$, as well as without the path from maternal abuse to novelty P300 amplitude $(\mathrm{AIC}=5008.402$, $\mathrm{BIC}=5169.703)$, and without the path from novelty P300 amplitude to self-report externalizing behavior $(\mathrm{AIC}=5011.274, \mathrm{BIC}=5172.576)$, respectively. The results showed that the final model itself has the smallest AIC and BIC. Taken together, novelty P300 amplitude partially mediated the relationship between maternal physical abuse and self-report externalizing behavior. The indirect effect accounts for $20.8 \%$ of the total effect between maternal physical abuse and self-report externalizing behavior.

\section{DISCUSSION}

This study tested the mediation hypothesis that the P300 ERP partly underlies the relationship between physical abuse and child externalizing behavior. Children who reported physical abuse by mothers also reported higher externalizing behavior problems and showed increased amplitude of the 


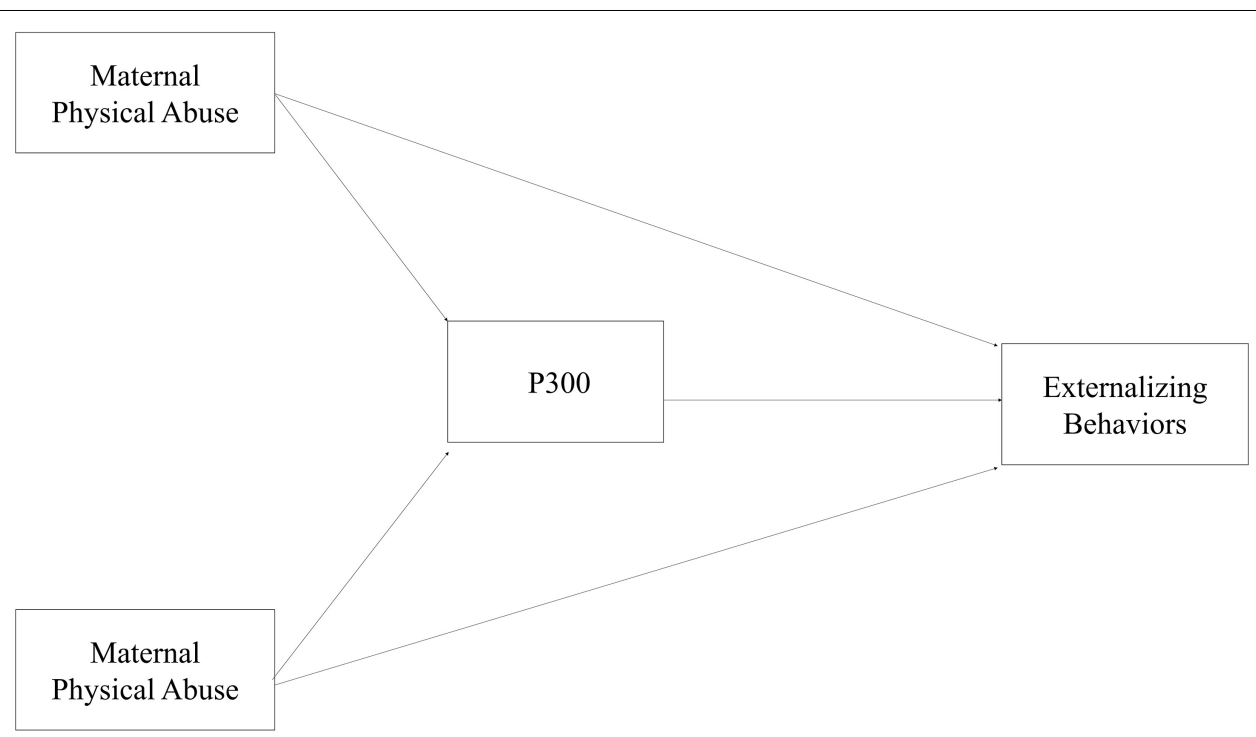

FIGURE 1 | The full mediation model. The model illustrates the relationship between maternal and paternal physical abuse and self-reported externalizing behaviors and the mediating role of P300. The variable P300 refers to any of the following variables: novelty P300 amplitude and target P300 amplitude. All the variables in the figure were measured at age 12. The model was adjusted child sex, socioeconomic status, area of residence, IQ at age 12 , anxiety at age 12 and externalizing behaviors in preschool at age 6.

TABLE 2 | Comparisons of behavior problems, performance on oddball task and P300 between physically abused children and their non-abused counterparts.

\begin{tabular}{|c|c|c|c|c|c|c|c|c|}
\hline & \multicolumn{2}{|c|}{ Maternal physical abuse } & \multirow[b]{2}{*}{$t / z$} & \multirow[b]{2}{*}{$p$} & \multicolumn{2}{|c|}{ Paternal physical abuse } & \multirow[b]{2}{*}{$t / z$} & \multirow[b]{2}{*}{$p$} \\
\hline & No $M \pm S D(M e d i a n)$ & Yes $M \pm S D$ (Median) & & & No $M \pm S D$ (Median) & Yes $M \pm S D$ (Median) & & \\
\hline Externalizing behavior at age 12 & $50.60 \pm 9.24$ & $56.43 \pm 14.24$ & 3.07 & $<0.001$ & $51.09 \pm 11.85$ & $55.18 \pm 10.15$ & 2.15 & 0.03 \\
\hline Externalizing behavior at age 6 & $12.84 \pm 6.91$ & $14.12 \pm 6.34$ & 1.02 & 0.31 & $12.68 \pm 6.54$ & $14.43 \pm 7.08$ & 1.40 & 0.17 \\
\hline Mean reaction time & $504.07 \pm 97.5$ & $482.64 \pm 78.09$ & 1.36 & 0.18 & $501.05 \pm 92.97$ & $489.56 \pm 90.25$ & 0.73 & 0.46 \\
\hline Correct response to target & $31.38 \pm 4.22(33)$ & $30.58 \pm 5.35(32)$ & $0.73^{w}$ & 0.46 & $31.32 \pm 4.1(32)$ & $30.74 \pm 5.48(33)$ & $0.11^{w}$ & 0.91 \\
\hline Errors to novels & $5.63 \pm 7.44(3)$ & $5.54 \pm 6.95(3)$ & $0.21^{w}$ & 0.83 & $6.16 \pm 8.09(3)$ & $4.55 \pm 5.25(3)$ & $0.23^{w}$ & 0.82 \\
\hline Errors to non-targets & $1.59 \pm 2.05(1)$ & $1.94 \pm 1.97(1)$ & $1.45^{w}$ & 0.15 & $1.47 \pm 1.81(1)$ & $2.15 \pm 2.34(1)$ & $1.88^{w}$ & 0.06 \\
\hline Novelty P300 amplitude ( $\mu$ V) & $9.63 \pm 4.47$ & $11.40 \pm 4.30$ & 2.35 & 0.02 & $10.04 \pm 4.66$ & $10.54 \pm 4.14$ & 0.67 & 0.51 \\
\hline Target P300 amplitude $(\mu \mathrm{V})$ & $10.98 \pm 7.13$ & $11.08 \pm 5.06$ & 0.09 & 0.93 & $11.31 \pm 7.15$ & $10.46 \pm 5.09$ & 0.77 & 0.44 \\
\hline
\end{tabular}

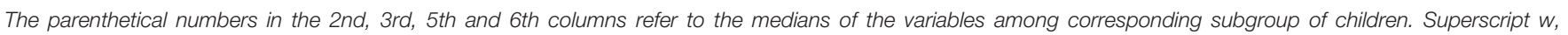
Wilcoxon rank-sum test.

novelty P300. Externalizing behavior was positively related to novelty P300 amplitude. Further path analysis revealed that enhanced P300 amplitude to novel stimuli partially mediated the relationship between maternal physical abuse and self-reported externalizing behavior after adjusting for child sex, socioeconomic status, area of residence, IQ, and earlier externalizing behavior. To our knowledge, findings appear to be the first to document the mediating role of P300 amplitudes on the abuse-externalizing relationship.

\section{The Relationships Among Physical Abuse, Novelty P300 Amplitude, and Externalizing Behaviors}

This study found increased novelty P300 amplitudes in a cognitive task using non-affective auditory stimuli among children who had experienced maternal physical abuse. This result is broadly consistent with prior findings showing that abused children are hypervigilant to negative visual and vocal stimuli (Pollak et al., 2001; Pollak and Tolley-schell, 2003; Shackman et al., 2007; McCrory et al., 2012; Gabriela et al., 2014; Shackman and Pollak, 2014; Okazaki et al., 2020). We extend prior literature by illustrating that physically abused children also showed hypervigilance to novel nonaffective stimuli. Taken together, physically abused children may demonstrate generalized hypervigilance and tend to orient more attentional resources involuntarily toward distractors or negative emotional stimuli in the environment. This feature may reflect an adaptive mechanism for abused children to be more capable of detecting, and hence reacting more efficiently to potential social threats in the environment (Pollak, 2015).

Further, the enhanced novelty P300 amplitude partially mediated the relationship between maternal physical abuse 
TABLE 3 | Pearson correlations between ERP and behavior problem.

\begin{tabular}{lcccc}
\hline & $\mathbf{1}$ & $\mathbf{2}$ & $\mathbf{3}$ & $\mathbf{4}$ \\
\hline 1. Externalizing behavior at age 12 & 1 & & & \\
2. Externalizing behavior at age 6 & $0.204^{\star}$ & 1 & & \\
3. Novelty P300 amplitude $(\mu \mathrm{V})$ & $0.255^{\star \star}$ & 0.171 & 1 & \\
4. Target P300 amplitude $(\mu \mathrm{V})$ & 0.028 & 0.135 & $0.250^{\star \star}$ & 1 \\
\hline
\end{tabular}

${ }^{*}, p<0.05 ;{ }^{* *}, p<0.01$.

and externalizing behaviors, which is generally consistent with the prior findings that attention problems mediated the relationship between physical abuse and aggression in children and adolescents (Kenny, 2016), and that attention bias toward mothers' angry faces or voices indicated by enhanced P300 amplitude mediated the association of physical abuse and anxiety (Debener et al., 2002). These data are consistent with the view that physical abuse affects the attention bias to novel cues that likely place them at increased risk for the development and maintenance of externalizing behavior (Liao et al., 2011).

However, the finding that increased novelty P300 was associated with more externalizing behaviors is contradictory to the existing findings of reduced novelty P300 responses of studies among criminals, offenders, and university students (Stanford and Kockler, 2007; Brazil et al., 2012; Venables and Patrick, 2014; Bernat et al., 2020). Nonetheless, some other studies among psychopaths reported no associations (Munro et al., 2007; Gao et al., 2011) or enhanced P300 to nonaffective auditory or digital stimuli (Raine, 1987; Raine and Venables, 1988; Roberti, 2004; Gao et al., 2018). Although differences in task modality (affective vs. non-affective) can be a possible reson for the mixed findings, it is also possible that different traits of psychopaths are related to different patterns of P300 change. This is supported by the notion proposed by Pasion et al. that P300 decrement may be a neurobiological marker of externalizing dispositions of many personality and mental disorders, whereas enhanced P300 amplitude during non-affective cognitive tasks is associated with interpersonalaffective psychopathic traits (Pasion et al., 2018). Hence, our finding of positive association of novelty P300 and externalizing behavior may suggest the interpersonal-affective impairment be the potential mechanism of the link between physical abuse and externalizing behavior. It may be also possible that this result was confounded by internalizing behaviors as externalizing and internalizing behaviors tend to be comorbid (Bernat et al., 2020).

It is worth noting that the participated children were generally healthy school children without obvious or diagnosed externalizing disorders, and, therefore, their neural activity may be different from those with diagnosed or severe antisocial behavior or psychopathy in the past studies. More longitudinal studies can be conducted to further investigate whether the neural activity toward novel stimuli changes from externalizing behavior to the course of externalizing disorders.

\section{Target P300 and Externalizing Behaviors}

Unlike the previous studies that reported reduced target P300 amplitude among individuals with generic antisocial behavior (Gao and Raine, 2009), substance abuse disorders (Iacono and Mcgue, 2002; Euser et al., 2012; Hamidovic and Wang, 2019), ADHD (Bitter, 2011), and conduct disorder (Iacono and Mcgue, 2002), we did not find a significant relationship between target P300 and externalizing behavior. It may be because the oddball task used in this study is not complex enough to reveal potential cognitive deficiency in such a sample of healthy children suggested by the finding that no significant difference in the behavioral responses in the oddball task was found between abused and non-abused children. Nonetheless, a recent meta-analysis did not find a significant mean effect of target-P300 (Go-P300) amplitude between individuals with and without ADHD (Kaiser et al., 2020). Bernat et al. (2020) found that lower novelty P300 amplitude elicited by a rotatedhead visual oddball task was associated with more externalizing behavior, but target P300 amplitude was not among a sample of healthy university students. Therefore, the exact relationship between target P300 and child externalizing problems needs further investigation.

\section{Maternal vs. Paternal Physical Abuse}

The present study did not find a significant association of paternal physical abuse with P300 and externalizing behavior in the path analysis, which is consistent with the previous findings. For example, our study among Chinese children using the same data source showed that in comparison to paternal physical abuse, maternal physical abuse showed

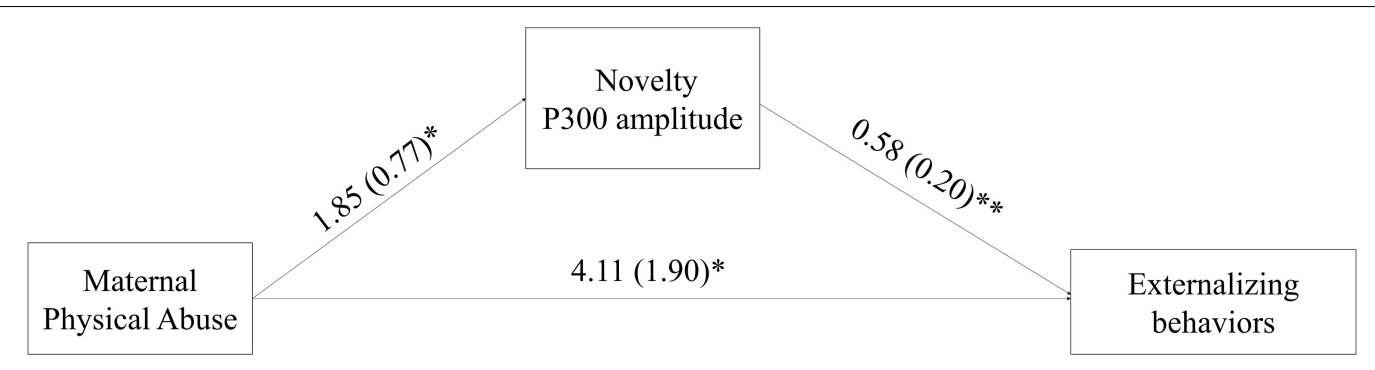

FIGURE 2 | The final mediation model with path coefficients. The model illustrates that novelty P300 amplitude mediated the relationship between maternal physical abuse and self-report externalizing behaviors. ${ }^{*}, p<0.05 ;{ }^{* *} p<0.01$. 
a more salient relationship with child externalizing and internalizing behaviors (Cui et al., 2018). Gao et al. (2010) reported that paternal care was not significantly associated with psychopathy after controlling for maternal care. Likewise, a meta-analysis by Kawabata et al. (2011) conducted a review of 48 studies and found that maternal parenting stress was associated with child relational regression, whereas paternal parenting stress was not.

Previous research suggested paternal and maternal abuse may link to externalizing behavior through different neurocognitive pathways that may be not captured by the P300 in the study. For example, Xing et al. (2018) found that inhibitory control mediated the relationship between maternal corporal punishment (CP) and child externalizing behavior, and working memory mediated the relationship between paternal CP and child externalizing behavior. More studies are needed to clarify the different effects of maternal and paternal parenting behaviors on child neurophysiological and behavioral development.

\section{Strengths and Limitations}

The study's strength lies in testing the mediating role of P300 on the relationship between physical maltreatment and externalizing behavior adjusting for earlier measured externalizing behavior in a relatively large sample $(N=155)$. The sample size is 3-5 times larger than the two prior studies $[N=30$ and 50 (Shackman et al., 2007; Shackman and Pollak, 2014)]. Nonetheless, causal inferences cannot be made as data was cross-sectional. Furthermore, a significant proportion of children of the original sub-cohort did not participate in the psychophysiological data collection. Although comparisons of the sociodemographic characteristics between the retained children and excluded children due to missing data or dropout from the parent study did not yield significant differences, potential differences in unobserved characteristics cannot be ruled out. In addition, other forms of child maltreatment were not assessed, and, therefore, the effect of physical abuse on P300 and externalizing behavior may be contaminated, especially considering that multiple forms of child maltreatment tend to co-occur (Brown et al., 2019). Also, this study examined the between group (exposure or non-exposure of physical abuse) differences, but did not examine the possible within group differences (e.g., frequency, severity, and chronicity) among children exposed to physical abuse in the past year. The physical abuse and externalizing behavior information were self-reported, and may be subject to report bias and common method bias. Last but not least, although the novel auditory oddball task is widely used in the area of behavioral problems with externalizing traits, it was relatively rarely used among maltreated children. It has been suggested that P300 elicited by different cognitive modalities can be different and such differences can be informative for distinguishing cognitive patterns across different populations (Barry et al., 2009; Nan et al., 2018). Future studies can utilize the bimodal/intermodal design of oddball task to further investigate the patterns of brain activity in the context of child maltreatment and externalizing behaviors.

\section{CONCLUSION}

In conclusion, physically abused children showed increased P300 amplitude to novel stimuli, and this enhancement partially mediated the relationship between physical abuse and externalizing behavior. The findings contribute to the very sparse literature on how psychophysiological pathways underpin the relationship between physical abuse and externalizing behavior. They further suggest that attention bias to novel/negative stimuli in the environment could be targeted to potentially treat childhood externalizing behavior associated with physical abuse. Such intervention studies could further test the casual nature of associations documented in this study. The findings also shed light on future research to investigate the mechanism using more rigorous methodology, such as measuring physical abuse and externalizing behaviors using objective or multipleinformant approach, taking other forms of child maltreatment into consideration, using other paradigms for ERP recording.

\section{DATA AVAILABILITY STATEMENT}

The raw data supporting the conclusions of this article will be made available by the authors, without undue reservation.

\section{ETHICS STATEMENT}

The studies involving human participants were reviewed and approved by the Institute Review Board of University of Pennsylvania and the Ethics Committee of Jintan Hospital. Written informed consent to participate in this study was provided by the participants' legal guardian/next of kin.

\section{AUTHOR CONTRIBUTIONS}

NC: conceived of the presented idea, performed the analysis, and wrote the manuscript. JL: conceptualized the cohort study, collected the data, conceived of the presented idea, and revised the manuscript. $\mathrm{AR}, \mathrm{CC}, \mathrm{TR}, \mathrm{AH}$, and $\mathrm{CM}$ : intellectual contribution to theorizing and revising the manuscript. All authors contributed to the article and approved the submitted version.

\section{FUNDING}

This study was supported by the National Institute of Environmental Health Sciences (grants R01-ES018858, K01ES015877, and K02-ES019878), the Research Award from Office of Nursing Research at the University of Pennsylvania School of Nursing, the National Social by the National Social Science Fund (18CSH061), the National Natural Science Fund (3210070458), and the Seed Fund for Candidates for "Qilu Outstanding Scholars" by Shandong University (2018WLJH46).

\section{ACKNOWLEDGMENTS}

We thank the child participants in the study. 


\section{REFERENCES}

Achenbach, T. M., and Rescorla, L. A. (2000). Manual for the ASEBA Preschool Forms \& Profiles. Burlington, VT: University of Vermont.

Barry, R. J., Clarke, A. R., McCarthy, R., Selikowitz, M., Brown, C. R., and Heaven, P. C. L. (2009). Event-related potentials in adults with attention-deficit/hyperactivity disorder: an investigation using an inter-modal auditory/visual oddball task. Int. J. Psychophysiol. 71, 124-131. doi: 10.1016/j. ijpsycho.2008.09.009

Bernat, E. M., Ellis, J. S., Bachman, M. D., and Hicks, B. M. (2020). P3 amplitude reductions are associated with shared variance between internalizing and externalizing psychopathology. Psychophysiology. 57:e13618.

Bitter, I. (2011). P300 deficits in adults with attention deficit hyperactivity disorder: a meta-analysis. Psychol. Med. 41, 1529-1538. doi: 10.1017/s0033291710001996

Brazil, I. A., Verkes, R. J., Brouns, B. H. J., Buitelaar, J. K., Bulten, B. H., and De Bruijn, E. R. A. (2012). Differentiating psychopathy from general antisociality using the P3 as a psychophysiological correlate of attentional allocation. PLoS One 7:e50339. doi: 10.1371/journal.pone.0050339

Brennan, G. M., and Baskin-Sommers, A. R. (2018). Brain-behavior relationships in externalizing: P3 amplitude reduction reflects deficient inhibitory control. Behav. Brain Res. 337, 70-79. doi: 10.1016/j.bbr.2017.09.045

Brown, S. M., Rienks, S., McCrae, J. S., and Watamura, S. E. (2019). The cooccurrence of adverse childhood experiences among children investigated for child maltreatment: a latent class analysis. Child Abuse Negl. 87, 18-27. doi: 10.1016/j.chiabu.2017.11.010

Bursac, Z., Gauss, C. H., Williams, D. K., and Hosmer, D. W. (2008). Purposeful selection of variables in logistic regression. Source Code Biol. Med. 3:17.

Chan, K. L. (2012). Comparison of parent and child reports on child maltreatment in a representative household sample in Hong Kong. J. Fam. Violence 27, 11-21. doi: $10.1007 / \mathrm{s} 10896-011-9405-1$

Cui, N., Deatrick, J. A., and Liu, J. (2018). Maternal and paternal physical abuse: unique and joint associations with child behavioral problems. Child Abuse Negl. 76, 524-532. doi: 10.1016/j.chiabu.2017.05.003

Cui, N., and Liu, J. (2016). Cognitive and behavioral risk factors for child physical abuse among Chinese children: a multiple-informant study. Child Adolesc. Psychiatry Ment. Health 10:36.

Cui, N., and Liu, J. (2020). Physical abuse, emotional abuse, and neglect and childhood behavior problems: a meta-analysis of studies in Mainland China. Trauma Violence Abuse 21, 206-224. doi: 10.1177/1524838018757750

Cui, N., Xue, J., Connolly, C. A., and Liu, J. (2016). Does the gender of parent or child matter in child maltreatment in China? Child Abuse Negl. 54, 1-9. doi: 10.1016/j.chiabu.2016.01.003

Dan, L., and Yu, J. (1990). Report on Shanghai norms for the Chinese translation of the Wechsler Intelligence Scale for children-Revised. Psychol. Rep. 67, 531-541. doi: 10.2466/pr0.67.6.531-541

Debener, S., Kranczioch, C., Herrmann, C. S., and Engel, A. K. (2002). Auditory novelty oddball allows reliable distinction of top - down and bottom - up processes of attention. Int. J. Psychophysiol. 46, 77-84. doi: 10.1016/s0167$8760(02) 00072-7$

Euser, A. S., Arends, L. R., Evans, B. E., Greaves-lord, K., Huizink, A. C., and Franken, I. H. A. (2012). The P300 event-related brain potential as a neurobiological endophenotype for substance use disorders: a meta-analytic investigation. Neurosci. Biobehav. Rev. 36, 572-603. doi: 10.1016/j.neubiorev. 2011.09.002

Fergusson, D. M., and Horwood, L. J. (2002). Male and female offending trajectories. Dev. Psychopathol. 14, 159-177. doi: 10.1017/s0954579402001098

Fry, D., Mccoy, A., and Swales, D. (2012). The consequences of maltreatment on children's lives: a systematic review of data from the East Asia and Pacific Region. Trauma Violence Abuse. 13, 219-233.

Gabriela, C., Ferreira, S., Crippa, J. A. S., and Osório, F. D. L. (2014). Facial emotion processing and recognition among maltreated children: a systematic literature review. Front. Psychol. 5:1460. doi: 10.3389/fpsyg.2014. 01460

Gao, Y., and Raine, A. (2009). P3 event-related potential impairments in antisocial and psychopathic individuals: a meta-analysis. Biol. Psychol. 82, 199-210. doi: 10.1016/j.biopsycho.2009.06.006

Gao, Y., Raine, A., Chan, F., Venables, P. H., and Mednick, S. A. (2010). Early maternal and paternal bonding, childhood physical abuse and adult psychopathic personality. Psychol. Med. 40, 1007-1016. doi: 10.1017/ s0033291709991279

Gao, Y., Raine, A., and Schug, R. A. (2011). P3 event-related potentials and childhood maltreatment in successful and unsuccessful psychopaths. Brain Cogn. 77, 176-182. doi: 10.1016/j.bandc.2011.06.010

Gao, Y., Zhang, W., Eisenbarth, H., Fung, A. L., Lu, M., Raine, A., et al. (2018). Personality and individual differences P3 amplitude and psychopathic traits in youths: distinct contributions of the grandiose-manipulative and daringimpulsivity traits. Pers. Individ. Differ. 120, 87-94. doi: 10.1016/j.paid.2017.08. 017

Gershoff, E. T., Lansford, J. E., Sexton, H. R., Davis-kean, P., and Sameroff, A. J. (2012). Longitudinal links between spanking and children's externalizing behaviors in a national sample of White, Black, Hispanic, and Asian American families. Child Dev. 83, 838-843. doi: 10.1111/j.1467-8624.2011.01732.x

Hallowell, E. S., Oshri, A., Liebel, S. W., Liu, S., Duda, B., Clark, U. S., et al. (2019). The mediating role of neural activity on the relationship between childhood maltreatment and impulsivity. Child Maltreat. 24, 389-399. doi: $10.1177 / 1077559519835975$

Hamidovic, A., and Wang, Y. (2019). The P300 in alcohol use disorder: a metaanalysis and meta-regression. Prog. Neuropsychopharmacol. Biol. Psychiatry 95:109716. doi: 10.1016/j.pnpbp.2019.109716

Iacono, W. G., and Mcgue, M. (2002). Minnesota twin family study. Twin Res. 5, 482-487. doi: 10.1375/136905202320906327

Kaiser, A., Aggensteiner, P. M., Baumeister, S., Holz, N. E., Banaschewski, T., and Brandeis, D. (2020). Earlier versus later cognitive event-related potentials (ERPs) in attention-deficit/hyperactivity disorder (ADHD): a metaanalysis. Neurosci. Biobehav. Rev. 112, 117-134. doi: 10.1016/j.neubiorev.2020. 01.019

Kallen, A. M., Perkins, E. R., Klawohn, J., and Hajcak, G. (2020). Cross-sectional and prospective associations of P300, RewP, and ADHD symptoms in female adolescents. Int. J. Psychophysiol. 158, 215-224. doi: 10.1016/j.ijpsycho.2020. 08.017

Kavanaugh, B. C., Dupont-frechette, J. A., Jerskey, B. A., Karen, A., and Kavanaugh, B. C. (2017). Neurocognitive deficits in children and adolescents following maltreatment: neurodevelopmental consequences and neuropsychological implications of traumatic stress. Appl. Neuropsychol. Child 6, 64-78. doi: 10. 1080/21622965.2015.1079712

Kawabata, Y., Alink, L. R. A., Tseng, W., Van Ijzendoorn, M. H., and Crick, N. R. (2011). Maternal and paternal parenting styles associated with relational aggression in children and adolescents: a conceptual analysis and meta-analytic review. Dev. Rev. 31, 240-278. doi: 10.1016/j.dr.2011.08.001

Kenny, D. (2016). Mediation. Avilable online at: http://davidakenny.net $/ \mathrm{cm} /$ mediate.htm.

Liao, M., Lee, A. S., Roberts-Lewis, A. C., Hong, J. S., and Jiao, K. (2011). Child maltreatment in China: an ecological review of the literature. Child. Youth Serv. Rev. 33, 1709-1719. doi: 10.1016/j.childyouth.2011.04.031

Liu, J., Cao, S., Chen, Z., Raine, A., Hanlon, A., Ai, Y., et al. (2015). Cohort profile update: the China Jintan child cohort study. Int. J. Epidemiol. 44:1548. doi: 10.1093/ije/dyv119

Liu, J., and Lynn, R. (2015). Chinese sex differences in intelligence: some new evidence. Pers. Individ. Dif. 75, 90-93. doi: 10.1016/j.paid.2014.11.002

Liu, J., Mccauley, L., Leung, P., Wang, B., Needleman, H., and Pinto-martin, J. (2011). Community-based participatory research (CBPR) approach to study children's health in China: experiences and reflections. Int. J. Nurs. Stud. 48, 904-913. doi: 10.1016/j.ijnurstu.2011.04.003

Liu, J., Mccauley, L. A., Zhao, Y., Zhang, H., and Pinto-martin, J. (2010). Cohort profile: the China Jintan child cohort study. Int. J. Epidemiol. 39, 668-674. doi: 10.1093/ije/dyp205

McCrory, E., De Brito, S. A., and Viding, E. (2012). The link between child abuse and psychopathology: a review of neurobiological and genetic research. J. R. Soc. Med. 105, 151-156. doi: 10.1258/jrsm.2011.110222

Muniz, C. N., Fox, B., Miley, L. N., Delisi, M., Cigarran, G. P., and Birnbaum, A. (2019). The effects of adverse childhood experiences on internalizing versus externalizing outcomes. Crim. Justice Behav. 46, 568-589. doi: 10.1177/ 0093854819826213

Munro, G. E. S., Dywan, J., Harris, G. T., Mckee, S., Unsal, A., and Segalowitz, S. J. (2007). Response inhibition in psychopathy: the frontal N2 and P3. Neurosci. Lett. 418, 149-153. doi: 10.1016/j.neulet.2007.03.017 
Nan, C., Wang, G., Wang, H., Wang, X., Liu, Z., Xiao, L., et al. (2018). The P300 component decreases in a bimodal oddball task in individuals with depression: an event-related potentials study. Clin. Neurophysiol. 129, 2525-2533. doi: 10.1016/j.clinph.2018.09.012

Okazaki, K., Ota, T., Makinodan, M., and Kishimoto, N. (2020). Associations of childhood experiences with event-related potentials in adults with autism spectrum disorder. Sci. Rep. 10:13447.

Pace, G. T., Lee, S. J., and Grogan-kaylor, A. (2019). Spanking and young children's socioemotional development in low- and middle-income countries. Child Abuse Negl. 88, 84-95. doi: 10.1016/j.chiabu.2018.11.003

Pasion, R., Fernandes, C., Pereira, M. R., and Barbosa, F. (2018). Neuroscience and biobehavioral reviews antisocial behaviour and psychopathy: uncovering the externalizing link in the P3 modulation. Neurosci. Biobehav. Rev. 91, 170-186. doi: 10.1016/j.neubiorev.2017.03.012

Patrick, C. J., Bernat, E. M., Malone, S. M., Iacono, W. G., Krueger, R. F., and Mcgue, M. (2006). P300 amplitude as an indicator of externalizing in adolescent males. Psychophysiology 43, 84-92. doi: 10.1111/j.1469-8986.2006. 00376.x

Polich, J. (2007). Updating P300: an integrative theory of P3a and P3b. Clin. Neurophysiol. 118, 2128-2148. doi: 10.1016/j.clinph.2007.04.019

Pollak, S. D. (2015). Multilevel developmental approaches to understanding the effects of child maltreatment: recent advances and future challenges. Dev. Psychopathol. 27, 1387-1397. doi: 10.1017/s0954579415000826

Pollak, S. D., Klorman, R., Thatcher, J. E., and Cicchetti, D. (2001). P3b reflects maltreated children's reactions to facial displays of emotion. Psychophysiology 38, 267-274. doi: 10.1111/1469-8986.3820267

Pollak, S. D., and Tolley-schell, S. A. (2003). Selective attention to facial emotion in physically abused children. J. Abnorm. Psychol. 112, 323-338. doi: 10.1037/ 0021-843x.112.3.323

Raine, A. (1987). Contingent negative variation, P3 evoked potentials, and antisocial behavior. Psychophysiology 24, 191-199. doi: 10.1111/j.1469-8986. 1987.tb00277.x

Raine, A., and Venables, P. H. (1988). Enhanced P3 evoked potentials and longer P3 recovery times in psychopaths.pdf. Psychophysiology 25, 30-38. doi: 10.1111/ j.1469-8986.1988.tb00954.x

Renner, L. M., and Boel-Studt, S. (2017). Physical family violence and externalizing and internalizing behaviors among children and adolescents. Am. J. Orthopsychiatry 87, 474-486. doi: 10.1037/ort0000260

Roberti, J. W. (2004). A review of behavioral and biological correlates of sensation seeking. J. Res. Pers. 38, 256-279. doi: 10.1016/s0092-6566(03)00067-9

Shackman, J. E., and Pollak, S. D. (2014). Impact of physical maltreatment on the regulation of negative affect and aggression. Dev. Psychopathol. 26, 1021-1033. doi: $10.1017 /$ s0954579414000546
Shackman, J. E., Shackman, A. J., and Pollak, S. D. (2007). Physical abuse amplifies attention to threat and increases anxiety in children. Emotion 7, 838-852. doi: 10.1037/1528-3542.7.4.838

Stanford, M. S., and Kockler, T. R. (2007). P3 amplitude reduction and executive function deficits in men convicted of spousal/partner abuse. Pers. Individ. Differ. 43, 365-375. doi: 10.1016/j.paid.2006.12.011

Straus, M. A. (2004). Prevalence of violence against dating partners by male and female university students worldwide. Violence Against Women 10, 790-811.

Straus, M. A., and Hamby, H. L. (1998). Identification of child maltreatment with the Parent-Child Conflict Tactics Scales: development and psychometric data for a national sample of American parents. Child Abuse Negl. 22, 249-270. doi: 10.1016/s0145-2134(97)00174-9

Su, L., Li, X., Luo, X., Wan, G., and Yang, Z. (1998). Re-validation of the Child Behavior Checklist and reliabilty and validity testing. Chin. J. Mental Health 12, 67-70.

Venables, N. C., and Patrick, C. J. (2014). Reconciling discrepant findings for P3 brain response in criminal psychopathy through reference to the concept of externalizing proneness. Psychophysiology 51, 427-436. doi: 10.1111/psyp. 12189

Wang, R., Wang, M., Gao, Y., Jiang, Y., Zhang, X., and Yao, S. (2013). Reliability and validity of the Chinese Version of Achenbach Youth Self-Report (2001 version). Chin. J. Clin. Psychol. 21, 977-980.

Xing, X., Wang, M., and Wang, Z. (2018). Parental corporal punishment in relation to children's executive function and externalizing behavior problems in China. Soc. Neurosci. 13, 184-189. doi: 10.1080/17470919.2016.1258009

Conflict of Interest: The authors declare that the research was conducted in the absence of any commercial or financial relationships that could be construed as a potential conflict of interest.

Publisher's Note: All claims expressed in this article are solely those of the authors and do not necessarily represent those of their affiliated organizations, or those of the publisher, the editors and the reviewers. Any product that may be evaluated in this article, or claim that may be made by its manufacturer, is not guaranteed or endorsed by the publisher.

Copyright (c) 2021 Cui, Raine, Connolly, Richmond, Hanlon, McDonald and Liu. This is an open-access article distributed under the terms of the Creative Commons Attribution License (CC BY). The use, distribution or reproduction in other forums is permitted, provided the original author(s) and the copyright owner(s) are credited and that the original publication in this journal is cited, in accordance with accepted academic practice. No use, distribution or reproduction is permitted which does not comply with these terms. 